\title{
An Empirical Investigation into Dual-Task Trade-offs while Driving and Dialing
}

\author{
Duncan P. Brumby \\ Department of Computer Science \\ Drexel University \\ Philadelphia, PA 19140 USA \\ Brumby@cs.drexel.edu
}

\author{
Dario D. Salvucci \\ Department of Computer Science \\ Drexel University \\ Philadelphia, PA 19140 USA \\ Salvucci@cs.drexel.edu
}

\author{
Andrew Howes \\ Manchester Business School \\ University of Manchester \\ Manchester, M15 6PB UK \\ HowesA@,Manchester.ac.uk
}

\begin{abstract}
Engaging in a secondary task, such as dialing a cell phone, while driving a car has been found to have a deleterious effect on driver performance. A point often overlooked though is that people can potentially vary the extent to which these two tasks are interleaved (i.e., attention can be returned to driving more or less often while dialing). To investigate this idea of strategic variability in multitasking behavior, an experiment was conducted in a driving simulator in which participants were instructed to focus on dialing as quickly as possible or on steering as safely as possible. It was found that participants drove more safely when encouraged to do so. However, driving safely necessarily brought about an increase in the total time to complete the dialing task because of frequent task interleaving. In contrast, there was a significant increase in the lateral deviation of the car from the lane centre when participants were encouraged to complete the dialing task as quickly as possible. These results suggest that contrary to existing advice, the total time that the driver is distracted is less important to safety than the strategy used for interleaving secondary and primary tasks. In particular, there may be value in designing mobile devices that facilitate short bursts of interaction for in-car use because allowing drivers to make additional glances back to the road while actively working on a concurrent secondary task might help to elevate some of the effects of distracted driving.
\end{abstract}

\section{Categories and Subject Descriptors}

H.5.2 [Information Interfaces and Presentation] User Interfaces: Evaluation/methodology; K.4.1 [Computers and Society] Public Policy Issues: Human safety; H.1.2 [Models and Principles] User/Machine Systems: Human factors.

\section{General Terms}

Experimentation, Human Factors.

\section{INTRODUCTION}

Mobile computing devices are increasingly ubiquitous in society with technology-rich environments replete with "infotainment" systems for work and pleasure. In many of these environments, interaction with such systems occurs while the person is performing another task. One multitasking environment that has garnered a great deal of attention is that

(C) Duncan Brumby, Dario Salvucci, \& Andrew Howes, 2007 Published by the British Computer Society

Volume 2 Proceedings of the 21 st BCS HCI Group Conference

HCI 2007, 3-7 September 2007, Lancaster University, UK Devina Ramduny-Ellis \& Dorothy Rachovides (Editors) occupied by the driver of a car. Many studies of driver distraction have painted a rich picture of how interaction with various secondary devices can impair performance (e.g., $[11,13])$, with by far the most attention given to distraction resulting from cellular phone use (e.g., $[1,8,10])$.

Because of the dangers of using a cell phone while driving, legislation has been introduced in many countries (including Australia, France, Germany, Japan, Russia, Singapore, and the UK; for a complete list see, [15]) banning drivers from using a handheld device while driving. Despite these legal deterrents, which often carry a substantial fine and other penalties, people continue to use their phones while driving. For instance, compliance with the UK ban has slipped from $90 \%$ from its introduction in 2003 to around $75 \%$ in 2007; that is, today there are some 10 million UK motorists who admit to using a phone while driving, even though this activity is against the law [14].

Given that it is difficult to make people stop engaging in secondary tasks while driving, there may be substantial value in developing a better understanding of how people multitask. For instance, this knowledge might be useful for providing guidance on how mobile devices might be better designed to make their use by the driver of a car less egregious. One proposed heuristic for secondary-task interaction states that it should be possible to complete a stand-alone task on an in-car device in less than 15 seconds [5]. This 15 -second rule, which is set out in a draft Society of Automotive Engineers standard [12] for car manufacturers and designers of in-car devices, is based on the logic that the more time a driver spends on a secondary task, the higher the risk of adversely affecting driver performance. However, some researchers, most notably Tijerina et al. [13], have argued that the 15 -second rule is a poor predictor of driver distraction effects across a variety of tasks.

Cognitive modeling has provided researchers another way to better understand multitasking behavior. While recent cognitive models have accounted for many performance measures of human driver behavior under single- and dual-task conditions $[8,9,10]$, these efforts have not attempted to capture possible strategic variability in multitasking behavior. To capture such strategic variability, Brumby, Howes, and Saluvucci $[2,3,4]$ have adopted a cognitive constraint modeling (CCM, [6]) approach. This CCM approach focuses on understanding the constraints on the interaction between the driver and the task environment, and allows for objective functions to represent desired trade-offs in relation to critical performance variables (e.g., tradeoffs between secondary-task time and driver performance).

In particular, Brumby, Howes, and Salvucci [2] consider the task of dialing a cell phone number while driving. In this task, a driver might dial all digits at once without returning attention to driving, or might dial digits singly, returning to driving after each digit. Between these two extremes exist a plethora of alternative ways to complete the task (i.e., whether to switch 
back to driving between each pair of key-presses). The model evaluated each strategy within this space of possible strategies making predictions for driver performance (lateral deviation) and total time to complete the dialing task. This analysis showed that while interleaving tasks more often might increase task time it should also lead to safer performance on the driving task because of the decrease in time between consecutive updates of steering control. Moreover, this analysis suggests that, contrary to the advice given above, the total time that the driver is distracted is less important to safety than the strategy used for completing a secondary task while driving.

Previous studies of driver distraction [1,8,10,11,13] have generally not attempted to understand possible strategic variability in human behavior while multitasking; therefore it is an open question whether people indeed exhibit the type of strategic variability in behavior explored by Brumby et al.'s $[2,3,4]$ modeling analysis. In this paper we present an experiment that was designed to investigate dual-task trade-offs while dialing a cell phone and steering a car. In particular, the aim of the study was to test the idea that changing the task objective from focusing on dialing as quickly as possible to focusing on steering as safely as possible should have effects on relevant task performance measures.

\section{METHOD}

\subsection{Participants}

Participants were eight students (two female) at Drexel University, aged between 20- and 32-years ( $M=23$-years). All participants were experienced drivers who had held a valid US driving license for at least 2 years, and who owned and regularly used a car. All participants also owned and regularly used a cell phone. Participants were paid $\$ 10$ for taking part in the experiment, which took approximately one hour.

\subsection{Materials}

\subsubsection{Driving Task and Setup}

The experiment was conducted in a fixed-base driving simulator at Drexel University. The simulator includes the front half of a Nissan 240sx with standard steering and pedal controls. These controls connect to a Macintosh desktop computer that runs the simulation and data collection software. The driving simulation was developed in The Open Racing Car Simulator (TORCS, available at http://torcs.sourceforge.net/) and was projected onto an 8 -foot wide screen in front of the simulator vehicle, resulting in a roughly $48^{\circ}$ field of view. The simulated driving environment used in the study was a simple highway-like environment, except that there were no other vehicles on the road. Drivers navigated the middle of a straight three-lane highway, where construction cones discouraged them from moving toward or into the outer two lanes.

The driving task required participants to control the steering of the car only; participants were not required to use the acceleration or brake pedals because the simulator maintained the car at a constant speed (described below). While this type of driving task is much simpler than a real-world driving environment, the motivation for focusing on steering control was two-fold. First, steering control directly affects lateral deviation, which serves as an important proxy for driving safety. Second, given normal speed control, drivers may slow down on their own accord while engaged in the dialing task. To control for this potential confound, we forced participants to drive at a particular speed so that the effect of driving speed on lateral deviation might be directly inferred.

\subsubsection{Dialing Task and Setup}

The dialing task used a Sony Ericsson Z710i phone. Hardware integration with the Macintosh desktop computer running the simulation and data collection software was achieved through a wireless Bluetooth connection. Software from Salling (available at http://www.salling.com/Clicker/) was used to develop an application to display experimental prompts, enter digits on the phone's screen, and send log event information to the experimental software running on the Macintosh desktop.

For the dialing task, participants entered a 10-digit sequence of numbers, which followed the typical structure of a North American phone number (i.e., $X X X-X X X-X X X X)$. This number entry task was preceded by a select key-press representing a "power-on" function and was followed by a second select keypress representing a "send" function, to initiate the call giving 12 key-presses in total. All participants entered the same phone number throughout the experiment. The number was based on a local Drexel University phone number (215-895$X X X X)$ so would be somewhat familiar to participants prior to the experiment. In this way, the aim was to simulate the situation where people might enter a familiar and often dialed phone number while driving.

\subsection{Experimental Design and Procedure}

At the beginning of the experiment, participants were given an overview of the experiment, in which they were informed that they would be required to dial a cell phone while driving a simulated car. A demonstration was given by the experimenter on how to use the cell phone; in particular, it was expressed to the participant that the phone number had to be entered correctly in order to end a trial and that a backspace key on the device could be used to delete incorrect digit entries from the phone's display.

Participants were given a practice period (approx. 5 minutes), in which they familiarized themselves with the device and practiced entering the phone number. In order to minimize the possible impact of learning effects on dual-task performance, participants entered the same phone number throughout the familiarization period. The main part of the study began once participants were well practiced at entering the number sequence correctly on the cell phone.

\subsubsection{Single-task conditions}

For the dialing task, participants completed two blocks of five trials under single-task conditions. Participants were instructed to enter the phone number as quickly and accurately as possible. A trial started when the participant pressed the select key on the phone. A trial ended only when the participant again pressed the select key after correctly entering the phone number. At the end of each trial, participants received feedback showing the time taken to enter the number (in seconds). At the end of a block of five trials, the average dial time for that block of trials was presented to the participant.

If an incorrect key-press was made, participants had to delete the incorrect digit from the cell phone's display, and then reenter the correct digit - only when the correct sequence of digits was entered would the phone number be accepted and the trial end. Moreover, feedback reflected the total time to complete the trial. In this way, participants were explicitly discouraged from making errors by designing the dialing task such that it had a built-in speed-accuracy trade-off - that is, making an error incurred a time cost in terms of deleting the incorrect key and re-entering the correct one. We found that this significantly reduced the number of errors made in dual-task conditions from those in an earlier pilot study, where participants were found to strategically make errors in order to terminate a trial where they lost control of the car while dialing. All error trials were later excluded from analysis (albeit unknown to the participant). 
After the participant had completed two blocks of single-task dialing, they were introduced to the driving task. Participants were informed that the driving task did not require them to use the accelerator or brake pedal and that the car would travel at a consistent speed. They were told that the aim of the task was to drive the car as close to the centre of the centre lane as possible. Participants were given a practice session to familiarize themselves with steering the car within the roadway.

Following the practice session, the driving task was performed at both a slow driving speed where participants drove the car at a constant speed of $35 \mathrm{mph}(\sim 56 \mathrm{kph})$ and also a fast driving speed where participants drove the car at a constant speed of 55 $\mathrm{mph}(\sim 87 \mathrm{kph})$. For each of the two driving speeds, participants completed two blocks of five trials. Participants were instructed to keep the car as close to lane centre as possible (minimizing lateral deviation) over a 10-second period of driving. At the end of each trial, participants received feedback information showing the root mean square error (RMSE) lateral deviation of the car from lane centre over the trial. Again, at the end of a block of five trials, the average lateral deviation for that block of trials was also presented. Finally, before the next trial could start, participants were notified by the experimental software to centre the car in the lane; the message persisted until the car was within $+/-0.20$ meters from the lane centre, ensuring that the car was in a central location at the start of each trial.

\subsubsection{Dual-task conditions}

For dual-task conditions the structure of the dialing task and the driving task remained the same as that described above. The only difference was that once the car was close to lane centre, the participant started a dual-task trial by pressing the select key on the phone. The trial ended when the participant pressed the select key again after correctly entering the phone number.

The primary aim of the experiment was to determine the consequences of varying task objective and driving speed in dual-task condition for dial time and lateral deviation. The experiment followed a $2 \times 2 \times 4$ (task objective $\mathrm{x}$ driving speed $\mathrm{x}$ trial block) completely within-subjects design. In order to manipulate the task objective, participants were given instructions to focus on either completing the dialing task as quickly as possible (minimizing dial time) or keeping the car as close to the centre of the lane as possible (minimizing lateral deviation). At the end of a given trial, participants received feedback aimed at emphasizing the focus performance variable (i.e., showing the dial time or lateral deviation achieved for that trial; note that feedback was not provided for the non-focus performance variable). In order that a participant adjusts to a particular task objective, conditions were blocked together, such that a participant completed a series of 40 dual-task trials where they focused on the dialing task and then completed a series of 40 dual-task trials where they focused on the steering task. The order in which each condition was completed was counterbalanced between participants, such that half of the participants in the study focused on minimizing dial time and then focused on minimized the cars lateral deviation, and viceversa for the other half of participants.

The experiment also manipulated driving speed. This manipulation was again counter-balanced between participants, such that within each task objective condition, half of the participants completed the slow speed $(35 \mathrm{mph})$ condition before the fast speed $(55 \mathrm{mph})$ condition, and vice-versa for the other half of participants. Finally, participants completed four blocks of five trials of each of the dual-task experimental conditions described above. In total, participants completed 80 dual-task trials ( 5 trials $\times 4$ blocks $\times 2$ task objective $\mathrm{x} 2$ speeds of car), where each condition was partially counter-balanced between participants.

\section{RESULTS}

Analysis focused on performance between the different dualconditions. In particular, we were interested in the time taken to correctly dial the phone number and RMSE lateral deviation of the car from the centre of the lane. For statistical analysis a $2 \times 2$ (task objective $\mathrm{x}$ car speed) repeated-measures ANOVA was used. (Due to space limitations we do not present an analysis of differences in performance over each block of trials.) As a point of contrast, we also present dual-task performance in the context of single-task performance.

Relatively few trials were excluded from the analysis because of participant error on the dialing task. Recall that participants were discouraged from making an error on the dialing task because correcting it incurred additional time costs. From a total of 160 single-task dialing trials, only $4(2.5 \%)$ were excluded because of error; from a total of 640 dual-task trials, $37(5.78 \%)$ trials were excluded because of error. These low error rates suggest that participants were competent users of the cell phone and also learnt the number during the familiarization period prior to the study.

Figure 1 shows the time to enter the phone number in singletask and dual-task conditions under varying task objectives. It can be seen in the figure that participants adjusted their dualtask strategy dependent on the task objective - when participants focused on the dialing task, dial time in the dualtask condition was more or less equivalent to dial time in the single-task condition. Whereas, when participants focused on the steering task, dialing time was slower in the dual-task condition than in the single-task condition. In dual-task conditions, dialing time was significantly faster when the objective was to focus on the dialing task than when the objective was to focus on the steering task, $F(1,7)=25.83$, $p<.001, M S E=2.65$. Driving speed did not have a significant effect on dial time, $F(1,7)=3.33, p=.11, M S E=.80$, nor was there a significant interaction, $F(1,7)=2.66, p=.15, M S E=.44$.

Figure 2 shows the RMSE lateral deviation of the vehicle for single-task and dual-task conditions under varying task objectives and driving speeds. It is clear from the figure that driving speed had an effect on lateral deviation. In the singletask condition, lateral deviation from lane centre was less when the car was traveling at a slow speed than at a fast speed, $t(7)=5.41, p<.001$. There was a similar main effect of driving speed on lateral deviation in dual-task conditions, $F(1,7)=35.06, p<.001, M S E=.01$.

While it is clear from Figure 2 that participants adjusted their dual-task strategy based on the task objective, the most interesting aspect of the data is the clear interaction effect between task objective and driving speed on lateral deviation in dual-task conditions. In particular, when participants focused on the driving task, lateral deviation in the dual-task condition was more or less equivalent to that in the single-task condition. Whereas, when participants focused on the dialing task, lateral deviation was greater in the dual-task condition than in the single-task condition, but only at the faster driving speed.

Statistical analysis found a main effect of task objective on lateral deviation: Lateral deviation was greater when the objective was to focus on the dialing task compared to when it was to focus on the steering task, $F(1,7)=5.04, p=.06, M S E=.01$. But there was also a significant trend for the task objective $\mathrm{x}$ driving speed interaction effect, $F(1,7)=3.64, p=.09, M S E=.01$. Follow-up tests of the simple effects of task objective at each driving speed showed that when driving at a fast speed lateral deviation was greater when participants focused on the dialing task rather than the steering task, $F(1,7)=5.30, p=.05$. However, at a slow driving speed there was not a significant simple effect of task objective on lateral deviation, $F(1,7)=.002, p=.96$. 


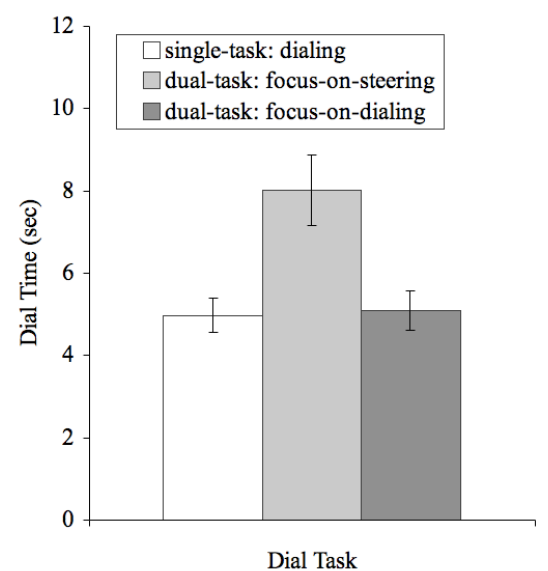

Figure 1: Time to enter the phone number in single-task and dual-task conditions under varying task objectives. Error bars represent $95 \%$ confidence intervals. (Dial-time is averaged over slow and fast driving speeds, because there was no effect of driving speed on dialing time.)

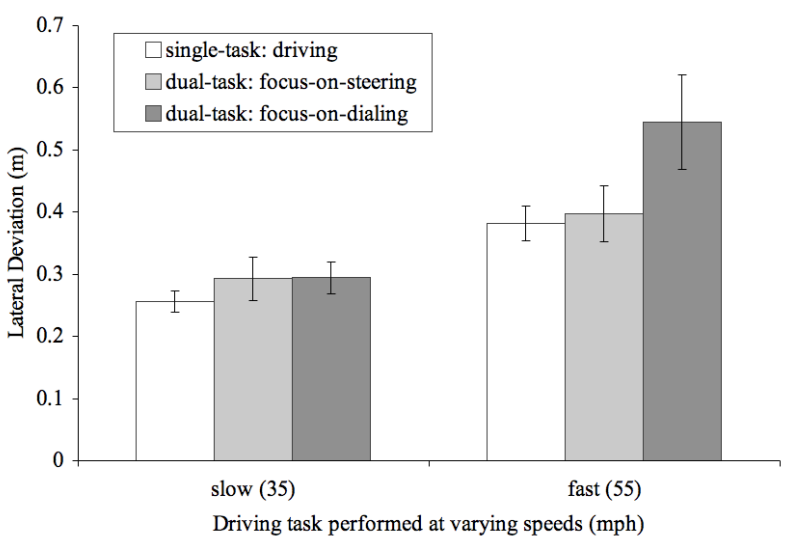

Figure 2: RMSE lateral deviation in single-task and dualtask conditions under varying task objectives and driving speeds. Error bars represent $\mathbf{9 5 \%}$ confidence intervals.

\section{DISCUSSION}

We have presented preliminary results from an experiment that investigated dual-task trade-offs while driving and dialing. These results clearly demonstrate that under dual-task conditions people can adjust their strategy dependent on varying task objectives but that this necessarily has consequences for the other tasks performance. When participants were instructed to focus on minimizing dial time, they dialed the number as fast as when entering it while not driving. When participants were instructed to focus on minimizing the lateral deviation of the car from the lane centre, they steered the car just as well as when they were not distracted by the dialing task. These strategy shifts had consequences for the other variable in dual-task conditions: Dial-time increased when participants focused on steering, and lateral deviation increased when participants focused on dialing (though only when the car was being driven at a faster speed). These data support the idea of psychological bottlenecks that limit the degree of parallelism between performing multiple tasks while driving (see also, [7]).

Implications can be drawn from the results of the study presented here for the design of mobile devices that could be used by the driver of a car. Given that it is difficult to make people stop engaging in secondary tasks while driving, efforts might be directed towards understanding how to better design mobile devices to make their use by a driver less dangerous. While a common metric for determining secondary-task safety is the total time it takes to complete a task on the device [5,12], our results suggest instead that there is value in simply encouraging drivers to pay greater attention to how they are driving while performing a secondary task. The total time that the driver is distracted is less important than the extent to which the driver is encouraged to make quick glances back to the road while actively working on the secondary task. This suggests that designing mobile devices that facilitate short bursts of interaction as opposed to requiring long stretches of interaction help to alleviate the effects of distracted driving.

\section{ACKNOWLEDGMENTS}

This research was supported by National Science Foundation grant \#IIS-0426674. We would like to thank Dan Markley and Mark Zuber for their exquisite hacking of the TORCS software and integration of the cell phone using Salling software. We also thank the four anonymous reviewers for providing comments for improving this paper.

\section{REFERENCES}

[1] Briem, V., \& Hedman, L.R. (1995). Behavioral effects of mobile telephone use during simulated driving. Ergonomics, 38, 25362562.

[2] Brumby, D.P., Howes, A., \& Salvucci, D.D. (2007). A cognitive constraint model of dual-task trade-offs in a highly dynamic driving task. In Proceedings of the SIGCHI Conference on Human Factors in Computing Systems (CHI 2007) (pp. 233-242). New York, NY: ACM Press.

[3] Brumby, D.P., Salvucci, D.D., \& Howes, A. (2007). Dialing while driving? A bounded rational analysis of concurrent multi-task behavior. To appear in Proceedings of the 8th International Conference on Cognitive Modeling. Ann Arbor, MI.

[4] Brumby, D.P., Salvucci, D.D., Mankowski, W., \& Howes, A. (2007). A cognitive constraint model of the effects of portable music-player use on driver performance. To appear in Proceedings of the Human Factors and Ergonomics Society 51st Annual Meeting. Santa Monica, CA: HFES.

[5] Green, P. (1999). The 15-Second Rule for Driver Information Systems. ITS America Ninth Annual Meeting Conference Proceedings, Washington, D.C.: ITS America.

[6] Howes, A., Vera, A., \& Lewis, R.L. (2007). Bounding rational analysis: Constraints on asymptotic performance. In W.D. Gray (Ed.) Integrated Models of Cognitive Systems (pp. 403-413). New York, NY: Oxford University Press.

[7] Levy, J., Pashler, H., \& Boer, E. (2006). Central interference in driving: Is there any stopping the psychological refractory period? Psychological Science, 17, 228-235.

[8] Salvucci, D.D. (2001). Predicting the effects of in-car interface use on driver performance: An integrated model approach. International Journal of Human-Computer Studies, 55, 85-107.

[9] Salvucci, D. D., \& Gray, R. (2004). A two-point visual control model of steering. Perception, 33, 1233-1248.

[10] Salvucci, D.D., \& Macuga, K.L. (2002). Predicting the effects of cellular-phone dialing on driver performance. Cognitive Systems Research, 3, 95-102.

[11] Salvucci, D.D., Markley, D., Zuber, M., \& Brumby, D.P. (2007). iPod distraction: Effects of portable music-player use on driver performance. In Proceedings of the SIGCHI Conference on Human Factors in Computing Systems (CHI 2007) (pp. 243-250). New York, NY: ACM Press.

[12] Society of Automotive Engineers (1998). SAE Standard for Navigation and Route Guidance Function Accessibility While Driving (SAE 2364), Committee Draft of November 23, Warrendale, PA: Society of Automotive Engineers.

[13] Tijerina, L., Johnston, S., Parmer, E., \& Winterbottom, M.D. (2000). Driver distraction with route guidance systems (Technical Report DOT HS 809-069). East Liberty, OH: NHTSA.

[14] "Careless talk", available at http://news.bbc.co.uk/2/hi/uk_news/ magazine/6382077.stm

[15] "Countries that ban cell phones while driving", available at http:// www.cellular-news.com/car bans/ 